M. Eddon

\title{
Fundamental Properties of Fundamental Properties
}

Oxford Studies in Metaphysics: Volume 8 (2013)

\section{Introduction}

Two grams mass and three coulombs charge are examples of quantitative properties. Such properties have certain structural features that other sorts of properties lack. How should we account for the distinctive structure of quantity? The answer to this question will depend, in large part, on one's other metaphysical commitments. In this paper I focus on the metaphysical framework offered by David Lewis.

I shall argue that, given the Lewisian framework, the most satisfying theory of quantity employs second-order relations. I then argue that the properties and relations invoked by a theory of quantity must be what Lewis calls perfectly natural, or fundamental. Together, these claims entail that there are perfectly natural second-order relations.

The thesis that there are perfectly natural second-order relations has an interesting consequence. The perfectly natural properties are generally taken to provide a minimal supervenience base for the qualitative facts. If we adopt perfectly natural second-order relations, however, then this is a mistake. The perfectly natural properties do indeed comprise a supervenience base, but this supervenience base is not minimal.

This paper proceeds as follows. In sections (2) and (3), I lay out some background assumptions, and sketch some of the structural features of quantity. In section (4), I assess several accounts of quantity, and argue that the one bestsuited to a Lewisian framework posits perfectly natural second-order relations. In section (5), I address worries that an account of the structural features of quantity, in terms of the perfectly natural, is not required. If such an account is not provided, I argue, then many accounts that make use of perfectly natural properties and relations are untenable. In section (6), I use the results of the previous sections to argue that the perfectly natural properties and relations do not comprise a minimal supervenience base.

\section{Background}

I will assume a broadly Lewisian framework.

I assume that we can quantify over possibilia, and I assume that possible individuals are world-bound. 
I assume that properties are abundant. ${ }^{1}$ For any set of possibilia, there is at least one property that applies to all and only the members of that set. Likewise, for any set of ordered $n$-tuples, there is at least one $n$-ary relation that applies to all and only the $n$-tuples in that set. ${ }^{2}$ Note that abundance extends to higher-order properties and relations as well. For every set of properties, there is a property that applies to all and only the members of that set. ${ }^{3}$

Every $n$-place function is associated with a set of ordered $n+1$-tuples, where the first $n$ elements are the arguments of the function, and last element is the function's output given those arguments. ${ }^{4}$ Since every function is associated with a set of ordered $n$-tuples, and every set of ordered $n$-tuples is associated with a relation, every function is associated with a corresponding relation. ${ }^{5}$

I assume that in addition to the abundant properties, there is a privileged set of perfectly natural properties that carve nature at the joints. The perfectly natural properties are, intuitively, those in virtue of which all else obtains; once we fix the distribution of perfectly natural properties, we fix everything else.

Perfectly natural properties figure prominently in a number of Lewis's analyses. For instance, two objects are duplicates iff they share all their perfectly natural properties, and their parts can be put into correspondence in such a way that corresponding parts have the same perfectly natural properties and stand in the same perfectly natural relations. ${ }^{6}$ Given duplication, we can analyze intrinsicality: a property $P$ is intrinsic iff for all duplicates, either both have $P$ or both lack it. ${ }^{7}$ And, in somewhat more involved ways, perfectly natural properties play key roles in Lewis's analysis of laws (see section (5.2)), counterfactuals (see section (5.3)), and causation (see section 5.4)).

\footnotetext{
${ }^{1}$ See Lewis ([1983] 1999, 9).

${ }^{2}$ I take no stand on whether properties and relations should be identified with sets of their instances. (See Eddon (2011) for reasons to think they should not.) Also, I ignore issues about whether properties should be identified (or associated) with sets as opposed to classes.

${ }^{3}$ See Lewis $(1986 b, 50)$ : "I do not want to restrict myself to properties of individuals alone; properties themselves have properties. Properties must therefore be sets so that they may be members of other sets."

${ }^{4}$ I.e. a function $f\left(x_{1}, \ldots, x_{n}\right)$ is associated with the set $\left\{\left\langle a_{1}, \ldots, a_{n}, f\left(a_{1}, \ldots, a_{n}\right)\right\rangle,\left\langle b_{1}, \ldots, b_{n}, f\left(b_{1}, \ldots, b_{n}\right)\right\rangle\right.$, $\ldots\}$.

${ }^{5}$ See Lewis (1991, 50-52) and Bigelow and Pargetter (1990, 45). I take no stand on whether functions should be identified (as opposed to just associated) with relations.

${ }^{6}$ See Lewis ([1983] 1999, 27) and Lewis $(1986 b, 61)$

${ }^{7}$ See Lewis $([1983] 1999,26)$ and Lewis $(1986 b, 62)$.
} 


\section{Quantities}

Among the perfectly natural properties are the quantitative properties posited by an ideal physics. ${ }^{8}$ These include properties such as two grams mass, three coulombs charge, $-1 / 2$ spin, and so on. ${ }^{9}$ (For simplicity, I restrict my attention to scalar quantities as opposed to, for instance, vector quantities.) Quantitative properties divide into kinds or "families"; e.g. all mass properties belong to the mass family, and all charge properties belong to the charge family, but no mass property belongs to the charge family and vice versa. Quantitative properties like these have distinctive features that qualitative properties - such as being a shoe do not. In particular, there are two characteristic features of quantitative properties:

Ordering. Quantitative properties of the same family can be ordered; e.g. two grams mass is less than three grams mass.

Distance. Quantitative properties of the same family stand in distance relations to one another; e.g. two grams mass and three grams mass are one gram mass apart. ${ }^{10}$

These features of quantitative properties are generally taken to be necessary. So, claims like the following are taken to be either necessarily true or necessarily false:

(1) two grams mass is less than three grams mass

(2) the distance between two grams mass and three grams mass is one gram

Call this the Necessity Assumption.

\section{Theories of Quantity}

Recall that properties are abundant: there is a property for every set of possibilia. Given this assumption, all rival accounts of quantity agree on which properties and relations exist. What they disagree on is which of these properties and relations play a role in grounding the structure of quantity. More precisely: they

\footnotetext{
${ }^{8}$ See Lewis $(1986 b, 60)$. Physics need not provide a complete inventory of the perfectly natural properties, but it plausibly provides at least a partial inventory.

${ }^{9}$ Denby (2001) argues that determinate properties such as these are fundamental rather than perfectly natural. In this paper, however, I use the terms interchangeably.

${ }^{10}$ For some discussion of these features of quantities, see Bigelow and Pargetter (1988), Ellis (1966, 24-38), Mundy (1987) and (1989), Swoyer (1987), inter alia.
} 
disagree on which belong to the elite set of perfectly natural properties and relations.

In this section, I explore several accounts of quantity that have been offered in the literature. ${ }^{11}$ I argue that the account of quantity best suited to the Lewisian framework employs second-order relations. A presupposition of this section is that some account must be provided. In the following section, I address those who reject this presupposition.

\subsection{Numerical Relations}

Consider my pen, which has 10 grams mass. One might take this at face value, and say that the number 10 literally plays a role in grounding this fact. So, one might say, the fact that my pen has 10 grams mass obtains in virtue of there being a perfectly natural relation - the mass-in-grams relation - that holds between my pen and the number 10 .

We now have a naive account of quantity: quantities are grounded in perfectly natural relations between objects and numbers. Call this the numerical relations theory. Suppose object $a$ has $2 \mathrm{~g}$ mass and object $b$ has $3 \mathrm{~g}$ mass. According to the numerical relations theory, there are no perfectly natural intrinsic mass properties that $a$ and $b$ instantiate. Rather, $a$ bears the perfectly natural mass-in-grams relation to the number 2 , and $b$ bears the perfectly natural mass-in-grams relation to the number 3 . And because the distance between the numbers 3 and 2 is 1 , the distance in mass between $a$ and $b$ is one gram. ${ }^{12}$

(A notational variant of the numerical relations theory has been proposed by Weatherson (2006). Weatherson argues that in place of perfectly natural determinate quantitative properties (like two grams mass), there are perfectly natural functions from objects to numbers, where a perfectly natural function is "such that there is some perfectly natural quantity such that for any $x, f(x)$ is the value that quantity takes with respect to $x . "(2006,488)$ Since a function is (identical to, or associated with) a set of ordered pairs, and a set of ordered pairs is

\footnotetext{
${ }^{11}$ The authors discussed do not always have an abundant conception of properties in mind (Armstrong, for instance, believes that properties are sparse), but nothing hangs on this.

${ }^{12}$ A more sophisticated variant of the numerical relations theory takes there to be a three-place perfectly natural relation holding between pairs of objects and numbers. For instance, if $a$ has two grams mass and $b$ has three grams mass, then there is a perfectly natural mass-ratio relation holding among $a, b$, and the number $2 / 3$. See Mundy (1988) for such a view. While this view is immune from the worry that choice of units is a matter of convention, the other worries for numerical relations theories raised in this section still apply.
} 
(identical to, or associated with) a relation, this amounts to the view that there are perfectly natural relations between objects and numbers.)

Numerical relations theories such as these appear to give a straightforward explanation of the distinctive structure of quantities: quantitative properties simply inherit the ordering and distance structure standardly associated with numbers. But such theories have significant drawbacks.

First, employing relations to numbers does not, by itself, yield a complete account of the structure of quantitative properties. For now, let us assume that numbers exist (see below for a discussion of dropping this assumption). The standard distance function, or metric, defined over numbers is one according to which the distance between any two numbers is the absolute value of their difference (i.e. $d(x, y)=|y-x|) \cdot{ }^{13}$ But this is not the only metric that may be defined over them. Another metric is the $p$-adic metric, according to which the distance between any two numbers is the $p$-adic norm of their difference. ${ }^{14}$ This metric yields the result that when the difference between two numbers is divisible by a large power of a prime number, the distance between them is small, and when the difference between them is divisible by a small power of a prime, the distance between them is large. ${ }^{15}$ Consider, for example, the 3-adic metric, which yields the following:

$$
\begin{aligned}
& d(3,5)=1 \\
& d(3,9)=1 / 3 \\
& d(3,84)=1 / 81
\end{aligned}
$$

Given the 3-adic metric, the distance between 3 and 5 is much greater than the distance between 3 and 84 .

Which of these metrics correctly captures the quantitative structure of properties like mass? Presumably, a proponent of this position will claim that it is the standard metric. But then we must say why the standard metric is the correct one to employ, and a $p$-adic metric is not.

One explanation is that the numbers themselves stand in ordering and distance relations to one another, some of which are more fundamental than others. The most fundamental capture the structure of the real numbers, and these are the ones that yield the standard metric over numbers. Other

\footnotetext{
${ }^{13}$ A metric over a set $S$ is a function $d: S \times S \rightarrow \mathbb{R}^{+}$such that, for all $x, y, z \in S$ :

(1) $d(x, y)=d(y, x)$ (symmetry)

(2) $d(x, y)=0$ iff $x=y$ (identity)

(3) $d(x, z) \leq d(x, y)+d(y, z)$ (triangle inequality)

${ }^{14} P$-adic norm: For any $p$-adic number $a / b \cdot p^{n}$, where $p$ is a prime number that does not divide either $a$ or $b,\left|a / b \cdot p^{n}\right|_{p}=1 / p^{n}$. P-adic metric: $d(x, y)=|y-x|_{p}$.

${ }^{15}$ See Holly (2001), inter alia.
} 
explanations may be explored as well. But in any case, numerical relations theories do not, by themselves, provide complete accounts of the structural features of quantity.

A second worry about numerical relations theories is that features that seem merely conventional may be construed as part of the fundamental structure of the world. Consider an object $c$ with $10 \mathrm{~g}$ mass. According to the numerical relations theory, $c$ bears the mass-in-grams relation to the number 10. But we could equally well have said that $c$ has .01 kilograms or .353 ounces. So in addition to bearing mass-in-grams to the number 10, $c$ bears mass-in-kilograms to the number .01 and mass-in-ounces to the number .353. Indeed, for any positive real number $n$, there is some scale of measurement according to which $c$ has $n$ units of mass - and thus for any $n, c$ bears some mass relation to $n$. Are all of these mass relations perfectly natural, or just one of them? If we choose one of these relations over the rest, we are left with the unhappy result that a certain scale of measurement is metaphysically privileged. This seems absurd - the units of measurement we use are purely a matter of convention. ${ }^{16}$ But if all of them are perfectly natural, then we are left with the unhappy result that there is massive redundancy in the perfectly natural properties. Moreover, we have no explanation for why so many of them march in lockstep.

These worries highlight an underlying methodological concern with the strategy of using numerical relations to account for the structural features of quantities like mass. We can represent the mass of an object using nearly any scale whatsoever - so choice of scale, and thus the number used to represent any given magnitude, should not enter into an account of the fundamental structure of quantity. To say that the fundamental facts about mass consist in relations to numbers is to confuse our representation of the fundamental facts with the facts themselves. ${ }^{17}$

A third reason to avoid numerical relations theories is a certain kind of methodological conservatism. The existence and structure of numbers are matters of considerable controversy, and it seems that a theory of quantity should

\footnotetext{
${ }^{16}$ For more discussion of conventions in measurement scales, see Ellis (1966, 74-86), Field (1980, 45), Hawthorne (2006), Milne (1986), and Mundy (1989).

${ }^{17}$ Ellis makes a similar observation when he warns against making inferences from the features of the representation of a property to the features of the property itself: "From the fact that the set of real positive numbers is everywhere dense, it does not follow that the sequence of heat states is everywhere dense. From the fact that this set of numbers is bounded below, but unbounded above, it does not follow that the set of heat states is bounded below and unbounded above. This is an important point, and we need to be constantly reminded of it, not only in connection with temperature measurement, but in connection with other kinds of measurement as well." $(1966,99)$
} 
not stand or fall depending on how those matters are resolved. For whether or not numbers exist, quantitative properties have structure that needs to be accounted for.

There are several reasons why numerical relations theories may initially seem promising. One reason is simplicity: such theories appear to give straightforward accounts of the structure of quantity. But as we've seen, they do not provide complete accounts of the structure of quantity. And complete extensions of them may be more complicated. Another reason is a desire to avoid unexplained necessary connections among properties. But if we hold that, say, the mass-in-grams and mass-in-ounces relations are both fundamental, then we still have unexplained necessary connections between these two relations. And if we say that only one of them is fundamental, then we avoid unexplained necessary connections at the cost of claiming that the fundamental structure of reality discriminates between grams and ounces. So while numerical relations theories may have some prima facie appeal, upon reflection these theories have little to recommend them.

\subsection{Intrinsic Structure of Quantitative Properties}

There is some intuitive pull to the thought that the ordering and distance relations the hold among quantitative properties are, in some sense, fixed by the intrinsic structure of the quantities themselves. D. M. Armstrong has proposed an account of quantity that develops this intuitive idea. According to Armstrong, quantitative properties are structural universals. A structural universal is a universal that has other universals as constituents, where constituency is a primitive relation analogous to mereological parthood. ${ }^{18}$ On Armstrong's account, every quantitative universal has an infinite number of "nested" constituent universals. Intuitively, the more constituents two quantitative universals share, the "closer" they are. For example, the five grams mass universal shares many constituents with the four grams mass universal, hence the property of five grams mass is close to the property of four grams mass. ${ }^{19}$

This structure succeeds in organizing quantities into families: mass universals have only other mass universals as constituents, charge universals have only other charge universals as constituents, etc. It also provides an ordering of

\footnotetext{
${ }^{18}$ Is the constituency relation between universals itself perfectly natural? If so, then this theory is technically a second-order theory, and therefore has the same surprising consequences as other second-order theories, For a defense of second-order theories of quantity (though not this theory in particular) see sections (4.4), (5), and (6).

${ }^{19}$ See Armstrong (1978, 116-131), (1988), and (1989, 102-107).
} 
quantities within families: the five grams mass universal has the four grams mass universal as a constituent, but not vice versa. But structural universals cannot accommodate the distance structure of quantitative properties. ${ }^{20}$

To see why, let's consider two different scenarios. Let's first suppose there is a possible world $w$ at which just a finite number of mass quantities are instantiated. ${ }^{21}$ Suppose there are only three objects with mass at $w$. Since Armstrong denies that there exist uninstantiated universals, it follows that there are only three mass universals $-a, b$ and $c-$ at $w$. Now suppose these universals have the following structure: $a$ has no constituents, $a$ is the only constituent of $b$, and $a$ and $b$ are the only constituents of $c$. On Armstrong's account, $b$ is equidistant from $a$ and $c$, no matter what mass values these universals correspond to. But if $a$ is the two grams mass universal, $b$ is the ten grams mass universal, and $c$ is the eleven grams mass universal, then $b$ is not equidistant from $a$ and $c$. The trouble is this: we've no guarantee that the gaps between the constituents of a universal are all the same "size." We've no guarantee that the distance between three grams mass and four grams mass is the same as the distance between four grams mass and five grams mass, or that the distance between two grams mass and ten grams mass is not the same as the distance between ten grams mass and eleven grams mass.

In order to ensure that the distance between two grams mass and ten grams mass is greater than that between ten grams mass and eleven grams mass, we must insist that the ten grams mass universal has other constituents besides the two grams mass universal - it also has nine grams mass, eight grams mass, etc. So let's do that - let's now suppose that it does have those mass universals as constituents. Indeed, let's suppose that there is a mass universal for every possible value of mass. (We can do this either by requiring the world to be such that, for every mass quantity, there is some thing at the world that instantiates that quantity, or by adopting a platonist conception of properties. Let us put aside worries about whether either of these constraints is plausible.)

Now we have a new problem. Since there are uncountably many possible values of mass, there are uncountably many mass quantities. So, every mass universal has an infinite number of constituents. But then every mass universal has the same number of constituents as every other mass universal - an infinite number. The four grams mass universal has as many universals in common with the three grams mass universal as it does with the 100 grams mass universal. Since

\footnotetext{
${ }^{20}$ Armstrong's account raises a number of other worries as well. See Eddon (2007) for a more detailed examination of this theory and its limitations.

${ }^{21}$ Armstrong would probably deny the possibility of such a world (see Armstrong 1988, 315).
} 
the extent to which universals share constituents determines the distance between values, the result is that the four grams mass universal is equidistant from three grams mass and 100 grams mass. But that is the wrong result.

In sum, the intrinsic structure of quantitative properties does not, by itself, fix all the structural features of quantity; we are still missing the relations of distance between quantitative properties. ${ }^{22}$ So let us try a different tack. The theories of quantity explored in the following sections have one feature in common: each invokes fundamental relations of some sort in order to account for the structure of quantities.

\subsection{First-Order Relations: Betweenness and Congruence}

One sort of relational account of quantity appeals to fundamental first-order relations. A nominalist version of such an account has been proposed by Hartry Field (1980). Since we are working within a realist framework, let us borrow Field's apparatus and sketch a realist counterpart of the theory.

On this account, there is a pair of perfectly natural, first-order relations corresponding to every family of quantitative properties. The first is a three-place betweenness relation, $a$ Bet $b c$, which can be intuitively understood as " $a$ is between $b$ and $c$." The second is a four-place congruence relation, $a b$ Cong $c d$, which can be intuitively understood as "the distance between $a$ and $b$ is congruent to the distance between $c$ and $d$." For example, suppose object $a$ has two grams mass, $b$ has three grams mass, $c$ has six grams mass, and $d$ has seven grams mass. Then $b$ is mass-between $a$ and $c$, or $a$ Mass-Bet $b c$; and $a$ and $b$ are masscongruent to $c$ and $d$, or $a b$ Mass-Cong $c d .^{23}$

\footnotetext{
${ }^{22}$ One might try to resuscitate Armstrong's account by replacing constituent universals with amphibians - entities capable of repeated occurrence as well as duplication (see Lewis [1986a] 1999, 98). Suppose there is a "smallest" unit of mass - say, one gram. Then, on this account, the universal two grams mass has two one gram mass amphibians as parts, and the universal three grams mass has three one gram mass amphibians as parts. The distance between two grams mass and three grams mass is determined by "counting up" the number of amphibians (see Wilson 2000 for an account of arithmetic that assimilates the counting (or natural) numbers with tally marks). But this attempt to rescue Armstrong's account is not successful. First, it's not clear that the notion of an amphibian is coherent. Second, putting aside the dubious status of amphibians, this account gets off the ground only if we assume that properties like mass are not continuous, which is false.

${ }^{23}$ While Field's account is generally presented using the betweenness predicate, it is more appropriate to replace this predicate with the two-place less than relation (LESS). This is because quantities like mass have a unique ordering, which cannot be captured using betweenness. Betweenness can be defined in terms of less than: $b$ is between $a$ and $c$ iff $a$ LESS $b$ LESS $c$ or $c$ LESS $b$ LESS $a$ (Field 1980, 56).
} 
There are three worries one might have with this sort of account. First: the representation and uniqueness theorems Field employs require certain assumptions. For instance, they require that the masses of the objects at any world be, intuitively, "evenly spaced." (If there is a $1 \mathrm{~g}$-mass object, a $2 \mathrm{~g}$-mass object, and a $5 \mathrm{~g}$-mass object, there must also be a $3 \mathrm{~g}$-mass object and a $4 \mathrm{~g}$-mass object. $)^{24}$ But that seems implausible - indeed, it's not obvious that the actual world satisfies this assumption. ${ }^{25}$

Second: adopting this account results in the conflation of intuitively distinct metaphysical possibilities. Consider a world $w$ like ours, but where all the quantities are doubled in value. (For example, an object with two grams mass in our world has four grams mass in $w$.) Because the ratios between quantities remain exactly the same, every claim about the betweenness and congruence relations that is true at our world is also true at $w$, and vice versa. But then what is the difference between $w$ and the actual world? If all we have are intra-world betweenness and congruence relations, then we cannot accommodate the intuition that $w$ is qualitatively distinct from the actual world. ${ }^{26}$

Third: if we adopt this sort of account, then we lose much of the utility of perfectly natural properties. Consider duplication: two things are duplicates iff they have the same perfectly natural properties, and their parts bear the same perfectly natural relations to one another. When combined with Field's account of quantity, this definition yields some unwelcome results. Suppose all my perfectly natural properties and relations are quantitative, so that all of my intrinsic properties supervene on the betweenness and congruence relations holding between my parts. Then any possible individual that preserves these betweenness and congruence relations counts as my duplicate, even if it is, intuitively, twice as massive or half the size.

Now, one might deny that this intuition should hold much weight, and thus deny that any duplicate of me really is twice as massive or half the size. But in many cases this tactic is implausible. Consider a world at which one of these possible individuals, call it Duplicate ${ }_{1}$, coexists with another, call it Duplicate ${ }_{2}$,

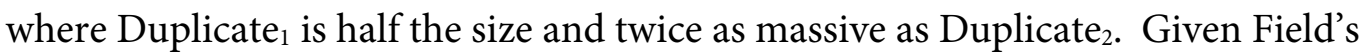
account, each is a duplicate of the other. And this is so even though the Fieldian

\footnotetext{
${ }^{24}$ This is the "solvability condition" given by Axiom 5 of Definition 8: if $c d \preceq a b$, then there exists $d^{\prime}$ between $a$ and $b$ such that $a d^{\prime}$ CONG $c d$ (Krantz et al. 1971, 172-173).

${ }^{25}$ See Hawthorne (2006), Liggins (2003), Melia (1998), Meyer (2009), and Mundy (1987, 32) and (1989) for some discussion of this and related objections.

${ }^{26}$ Though see Dasgupta (2013) for an attempt at accommodating this intuition without appealing to cross-world betweenness and congruence relations.
} 


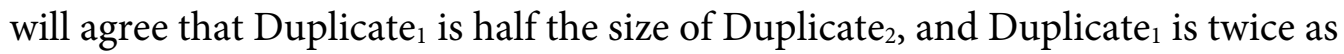
massive as Duplicate ${ }_{2}$. These implausible implications will filter into any account that makes use of a notion of duplication - including intrinsicality, supervenience, determinism, and so on.

Of the three worries raised above, the first two may be mitigated by adopting an ontology of possible worlds. The betweenness and congruence relations may then hold among possibilia in different possible worlds, guaranteeing that we have the objects needed to ground the intuitively correct numerical representation of quantitative properties. But this move only exacerbates the third worry - that we lose much of the theoretical utility of the perfectly natural properties.

We've already seen that difficulties arise for duplication. And when duplication goes awry, so does much else. Consider the thesis of determinism: on Lewis's characterization, laws of nature $L$ are deterministic iff among the worlds where $L$ holds, any two worlds that have duplicate initial temporal segments are duplicates simpliciter. ${ }^{27}$ Now consider two worlds, $w_{1}$ and $w_{2}$, alike in their laws of nature. World $w_{1}$ contains just three point-sized objects, all with the same mass. World $w_{2}$ is, intuitively, exactly the same as $w_{1}$ until time $t$. At $t$, the masses of the objects in both worlds increase, but the masses of the objects at $w_{2}$ increase twice as much as the masses of the corresponding objects at $w_{1}$. Worlds $w_{1}$ and $w_{2}$ are exactly alike with respect to their intra-world patterns of betweenness and congruence. By definition, they are duplicates. Therefore, $w_{1}$ and $w_{2}$ do not constitute a counterexample to the claim that the laws of nature at $w_{1}$ and $w_{2}$ are deterministic. But this seems like the wrong result.

On Field's original account, where betweenness and congruence relations hold only between individuals located at the same world, one could resist this result by denying that worlds $w_{1}$ and $w_{2}$ are distinct. But on the account under consideration, the Fieldian does recognize $w_{1}$ and $w_{2}$ as distinct possibilities, because these worlds differ in their inter-world relations. And so this move is not available.

Theses formulated in terms of supervenience also cause trouble. Consider the following supervenience claim: the distribution of mental properties supervenes on the distribution of perfectly natural physical properties. Now consider a possible world $w$ that is a physical duplicate of our world, but where all the corresponding masses are doubled and all the corresponding charges are halved. Suppose that nothing is conscious at $w$. It seems that the existence of

${ }^{27}$ See Lewis ([1983] 1999, 31-32). 
such a world does not constitute a counterexample to physicalism, because the fundamental physical structure of $w$ is very different from the fundamental physical structure of the actual world. On Field's account, however, $w$ is a physical duplicate of our world, and thus $w$ is a counterexample to physicalism. That seems like the wrong result.

Again, on Field's original account, one could resist this result by denying that $w$ is distinct from the actual world (thereby denying that nothing is conscious in $w$ ). But on the account under consideration, the Fieldian does recognize $w$ as a possibility distinct from the actual world, since it differs from the actual world with respect to its inter-world relations. And similar worries arise with other supervenience claims as well.

Another example: the distribution of perfectly natural properties and relations are supposed to comprise a basis for characterizing a world exhaustively. Consider two worlds, $w_{1}$ and $w_{2}$, with the same distribution of perfectly natural properties and relations. The inter-world mass relations are such that parts of $w_{1}$ are twice as massive as corresponding parts of $w_{2}$. On the account under consideration, the proposition that inhabitants of $w_{1}$ are twice as massive as those of $w_{2}$ is true. So which quantitative facts obtain at a world is partly a matter of the relations their inhabitants bears to other-worldly things. In that case, it seems that the perfectly natural properties and relations do not suffice to characterize a world exhaustively, since they do not suffice to characterize a world's quantitative structure. Again, the original Fieldian could deny that $w_{1}$ and $w_{2}$ are distinct worlds, but the Fieldian who adopts cross-world betweenness and congruence relations cannot.

Given these unpalatable consequences of allowing cross-world betweenness and congruence relations, one might retreat to the original Fieldian view and claim that betweenness and congruence relations hold only among objects located at the same world. But given the broadly Lewisian framework we're assuming, it's not clear this is even an option. The axioms governing Field's system entail that betweenness is connected: for any $x, y$, and $z$ over which betweenness is defined, either $x$ Bet $y z$ or $y$ Bet $x z$ or $z$ Bet $x y \cdot{ }^{28}$ So consider three massive objects, $a, b$, and $c$, each located at a different world. The requirement that betweenness is connected entails that there is some mass-betweenness relation that holds among $a, b$, and $c$ - and so it entails that there are cross-world mass-betweenness relations. Moreover, this result isn't confined to mass; connectedness requires cross-world spatiotemporal-betweenness relations as well.

\footnotetext{
${ }^{28}$ See Krantz et al. $(1971,172)$.
} 
And this result conflicts with Lewis's requirement that possible worlds are spatiotemporally isolated from one another. ${ }^{29}$

Anyone who wishes to retain the utility of perfectly natural properties in these and other areas will not welcome these results. For those who would like to situate an account of quantity within the Lewisian framework, a Fieldian account of quantity holds little appeal.

\subsection{Second-Order Relations: Less than or equal to and Sum of}

Finally, let's turn to an account of quantity offered by Brent Mundy (1987). Mundy assumes a platonist conception of properties, according to which properties exist necessarily even if nothing instantiates them. Included among the first-order properties are quantities such as two grams mass. In addition, there are two perfectly natural second-order relations holding between the quantitative properties: a two-place relation less than or equal to $(\lessgtr)$ and a three-place relation sum of $\left(^{*}\right)$. The less than or equal to relation generates the ordering structure of quantity; for example, two grams mass $\lesssim$ three grams mass $\lesssim$ four grams mass, and so on. The sum of relation generates the distance structure of quantity; for example, the sum of two grams mass and three grams mass is five grams mass (i.e. *(two grams mass, three grams mass, five grams mass). ${ }^{30}$

This theory avoids the objections raised against the accounts of quantity considered earlier. It does not quantify over numbers, and so steers clear of the worries associated with numerical relations theories. It does not rely solely on the intrinsic quantitative properties, and so avoids the problems that beset purely non-relational accounts, such as Armstrong's. Finally, because it adopts a platonist conception of properties, it avoids many of the counterintuitive implications of first-order relational theories, such as Field's. ${ }^{31}$ For these reasons, Mundy's account has significant advantages over its rivals. This gives us reason to prefer it. And since Mundy's account makes use of perfectly natural second-order relations, this gives us reason to admit perfectly natural second-order relations. ${ }^{32}$

\footnotetext{
${ }^{29}$ See Lewis $(1986 b, 69-81)$.

${ }^{30}$ See Mundy $(1987,37-38)$ for the formal presentation.

${ }^{31}$ See Mundy $(1987,32)$ for comparisons of first and second-order theories.

${ }^{32}$ Another second-order account has been proposed by Bigelow and Pargetter (1988) and (1990). In place of first-order quantitative properties, they posit first-order relations like twice as massive as and half as long as. And in place of the second-order relations $\lesssim$ and *, they posit second-order "relations of proportion." These second-order relations are intended to "impose an ordering" on the first-order relations, "explain[ing] how one thing can be closer to a second in, say, mass than it is to a third." $(1990,59)$ For example, it seems we want to say that the relation twice as massive as is "bigger" than the relation half as massive as; indeed, twice as massive as is necessarily "four times
} 
(There is a first-order version of this view, which might be more agreeable to those squeamish about higher-order properties and relations. Call this the Squashed Mundy View. On the Squashed Mundy View, the second-order relations $\lesssim$ and ${ }^{\star}$ are replaced with family-specific first-order relations. So, for instance, there is a perfectly natural first-order relation mass less than to equal to $\left(\varsigma_{\mathrm{m}}\right)$, and a perfectly natural first-order relation mass sum of $\left({ }^{*}{ }_{\mathrm{m}}\right)$. Now, suppose object $a$ instantiates two grams mass, $b$ instantiates three grams mass, and $c$ instantiates five grams mass. On the Squashed Mundy View, $a \nwarrow_{\mathrm{m}} b \nwarrow_{\mathrm{m}} c$, and $c$ is the mass sum of $a$ and $b$ (i.e. ${ }_{\mathrm{m}}(a, b, c)$ ). While this view avoids positing perfectly natural second-order relations, it requires that objects in different worlds stand in perfectly natural relations to one another. ${ }^{33}$ And any objection against the original Mundy view on the grounds that the relations it posits are redundant, and hence not perfectly natural (see section (5)), applies equally well to the Squashed Mundy View. Thus a Lewisian has little reason to prefer the Squashed Mundy View to the original.)

\section{Perfectly Natural Second-Order Relations}

Let's take stock. I have argued that the account of quantity best suited to a Lewisian framework appeals to perfectly natural second-order relations. Still, there are a few reasons one may doubt that perfectly natural relations of this sort are really required.

One might argue that because these second-order relations supervene on the distribution of first-order quantitative properties, we get them "for free." Since these relations are necessary, ${ }^{34}$ they trivially supervene on any property whatsoever (a fortiori they supervene on the first-order quantitative properties),

\footnotetext{
bigger" than half as massive as. And this is what the second-order relations of proportion are supposed to capture. The trouble with this account is that it is not clear how the second-order relations of proportion that Bigelow and Pargetter posit could capture the ordering and distance structure of quantity. Very roughly, the worry is that these second-order relations do not impose any axiomatic constraints on the instantiation pattern of first-order relations, and so do nothing to help capture the structural features of quantity (see Eddon forthcoming and Forge 1995).

In any case, even if one is unpersuaded by this criticism, the central moral of the paper applies: Bigelow and Pargetter's theory employs second-order necessary relations that must be construed as perfectly natural (as I argue in section (5)), which in turn entails that the perfectly natural properties do not comprise a minimal supervenience base (as I argue in section (6)).

${ }^{33}$ Thanks to Boris Kment here.

${ }^{34}$ The relevant notion of a "necessary relation" coincides with Lewis's definition of an internal relation: $R(x, y)$ is internal iff for any possible duplicate of $x, x^{\prime}$, and any possible duplicate of $y, y^{\prime}$, $R(x, y)$ iff $R\left(x^{\prime}, y\right)$ (Lewis $(1986 b, 62)$. I assume that properties exist at every world, although they may not have instances at every world.
} 
and so they contribute only redundantly to any characterization of reality. Therefore, they do not belong to the elite set of perfectly natural properties and relations. Once we have the perfectly natural intrinsic quantitative properties, nothing more needs to be said about the ordering and distance relations that hold between them.

In a related vein, one might be reticent to posit an objective distinction between natural second-order relations and gerrymandered ones without evidence that such a distinction is required beyond an account of the structure of quantity. After all, a more conservative explanation for why we're inclined to posit such a distinction is that some second-order relations are interesting or useful to us, and nothing more. If the distinction between perfectly natural quantitative second-order relations and gerrymandered ones is not required to do any philosophical work, then it seems unduly extravagant to claim that certain second-order relations are metaphysically privileged.

In essence, both of these challenges deny that an account of the structural features of quantity is needed. In the following sections, I address these two skeptical challenges. I argue that unless the ordering and distance structure of quantity is taken to be perfectly natural, many accounts that make use of perfectly natural first-order properties are untenable. The same considerations weigh in favor of taking some second-order relations to carve reality at the joints, and so to contribute - non-redundantly - to a complete characterization of reality. In section (6), I discuss a consequence of the claim that these second-order relations are perfectly natural: the perfectly natural properties do not comprise a minimal supervenience base.

To what extent do these worries, and my replies, hang on the details of Mundy's account? The first worry applies to any account of quantity that employs necessary relations, while the second applies to any account of quantity whatsoever. My responses address both worries. For simplicity, I assume that Mundy's account is correct. But my defense of this account can be tailored to fit other accounts of quantity as well.

\subsection{Resemblance}

Because properties are abundant, there is always some gerrymandered property that any two things will share, similar or not. Consider two electrons, $e_{1}$ and $e_{2}$. These electrons share the property being $e_{1}$ or being $e_{2}$. They also share the property of having $1.6 \times 10^{-19}$ coulombs charge. But only the latter property captures an objective similarity between the two. 
So the sharing of some properties makes for genuine resemblance, and the sharing of others does not. How can we distinguish between them? Enter natural properties: when two things share a perfectly natural property, they genuinely resemble one another in some respect. ${ }^{35}$

Quantitative properties cause trouble for this analysis. Suppose we have three objects $-a, b$, and $c$. Each instantiates a different amount of mass, and none instantiates any other perfectly natural property. It seems that the degree to which they objectively resemble each other is given by the distances between their mass quantities. Suppose $a$ instantiates $.001 \mathrm{~g}$ mass, $b$ instantiates $.002 \mathrm{~g}$ mass, and $c$ instantiates 5000 metric tons of mass. Which of these is least like the others? The answer seems to be $c .^{36}$

Yet the characterization of resemblance as the sharing of natural properties will not help us here. Objects $a, b$, and $c$ instantiate different quantities of mass, and so they do not share any perfectly natural properties. We might posit another fundamental property - the determinable mass - which $a, b$, and $c$ all instantiate, and use this to capture the fact that they resemble one another. But this won't help us capture degrees of resemblance - for instance, that $b$ resembles $a$ more closely than $c$.

Nor will appealing to non-fundamental second-order relations help us. For among the first-order properties, there are myriad second-order distance relations, each of which delivers a different judgment as to which objects are more similar. For instance, there are second-order relations corresponding to "discrete metrics," such that every mass quantity is equidistant from every other one (and zero distance from itself). There are second-order relations corresponding to "doubling metrics," such that the distance between, say, three grams mass and four grams mass is double the distance between two grams mass and three grams mass, which is double the distance between one gram mass and two grams mass. There are second-order relations corresponding to "switchy metrics" that effectively switch the "position" of some of the quantities, so that, say, three grams mass is closer to 500 grams mass than to two grams mass. And so on. If we want to capture the intuition that objects $a$ and $b$ resemble each other more than either resembles $c$, we need to say that one of these relations is privileged, or perfectly natural.

At this stage, one might wonder whether these intuitions about the resemblance of quantities should be taken so seriously. Even if two grams mass is

\footnotetext{
${ }^{35}$ See Lewis ([1983] 1999, 13) and $(1986 b, 60)$.

${ }^{36}$ See also Denby $(2001,299)$.
} 
closer to one gram mass than to 100 grams mass, it is far from obvious that there is a fact of the matter about whether two grams mass objectively resembles one gram mass more than 100 grams mass. But, as we'll see, this is just the tip of iceberg.

\subsection{Laws}

Let the best system of a world be the set of true sentences that provides, on balance, the simplest and most informative description of the distribution of the perfectly natural properties and relations at that world. On Lewis's analysis of laws, the laws at a world are the regularities entailed by the best system of the world.

But how shall we judge the simplicity and informativeness of candidate systems? We need to place some restrictions on the language they are stated in. Otherwise, a system may be maximally strong and simple, but only artificially so. Consider a language with the predicate $F$, where $F$ applies to all and only the things at the world in question. Then the system containing only the sentence "for all $x, x$ is an $F$ " will be maximally simple and maximally informative. This is not the result we want. ${ }^{37}$

Enter natural properties. If we require the best system to be expressed in a language whose predicates refer only to the perfectly natural properties and relations, then this language won't include predicates like $F$ and we avoid the worry above. So, the laws of nature are the regularities entailed by the system that, when stated in the language of natural properties, best balances simplicity and informativeness. ${ }^{38,39}$

\footnotetext{
${ }^{37}$ See Lewis ([1983] 1999, 42).

${ }^{38}$ See Lewis ([1983] 1999, 39-43) and ([1994] 1999, 231-232).

${ }^{39}$ One objection to the Lewisian analysis of laws is that the predicates appearing in standard formulations of the laws of physics do not express perfectly natural properties (see Hawthorne (2006, 236-237)). For instance, Newton's second law of motion $F=m a$ does not state that the determinable property force is literally identical to the determinable property resulting from "multiplying" mass by acceleration. "Equals," "multiplication", and other mathematical operations apply to numerals, not to properties. Rather, given the appropriate numerical representations of force, mass, and acceleration properties, $F=m a$ expresses a relationship between these values that reflects the nomic relationship between the determinate quantities. Exactly how the perfectly natural predicates enter into this representation is not a straightforward matter, and I will not address it here (see Denby (2001) for a proposal). I note only that however the matter is resolved, the problem raised in this section remains. For the standard formulations of the laws - the formulations used in assessing simplicity and informativeness - presuppose a numerical representation of quantitative properties that respects the perfectly natural second-order relations. If there are no perfectly natural second-order relations to respect, then there's no reason to use one formulation rather than another when assessing simplicity.
} 
Let's look more carefully at the criterion of simplicity. Consider a chaotic world where the trajectories of particles have few discernible patterns or regularities. Suppose that there are only three instances at which a (non-zero) force is applied to an object. In the first instance, an $8 \mathrm{~N}$ force is applied to a $1 \mathrm{~kg}$ particle, and the particle accelerates at a rate of $8 \mathrm{~m} / \mathrm{s}^{2}$. In the second instance, a $10 \mathrm{~N}$ force is applied to a $3 \mathrm{~kg}$ particle, and the particle accelerates at a rate of $.1 \mathrm{~m} / \mathrm{s}^{2}$. And in the third instance, a force of $2 \mathrm{~N}$ is applied to a $6 \mathrm{~kg}$ particle, and the particle accelerates at a rate of $4 /(3 \pi) \mathrm{m} / \mathrm{s}^{2}$. Now consider a candidate system of laws for this world that consists of only the following force law:

(L) $F=m a$ for all $m \leq 2$, and $F=(m+7)^{2} a$ for all $2<m<5$, and $F=(m \pi / 4) a$ for all $m \geq 5$

where we're employing the standard (intuitively correct) distance relations between mass quantities, and using a numerical representation of these relations that yields values in kilograms.

With respect to simplicity, a system consisting only of (L) does not appear to score very well. But now consider a metric function $d^{\prime}$ that differs from the standard one in the following way: for every quantity $m$ that the standard representation assigns a number $\leq 2, d^{\prime}$ assigns $m$, and for every quantity $m$ which the standard representation assigns a number between 2 and $5, d^{\prime}$ assigns $(m+$ $7)^{2}$, and for every quantity $m$ which the standard representation assigns a number $\geq 5, d^{\prime}$ assigns $m \pi / 4$. Given $d^{\prime}$, we can reformulate the law like this:

(L') $\quad F=m a$

Now the system looks much simpler.

When comparing the simplicity of the laws of this system to those of other candidate systems, which formulation should we use? Both are stated in the language of natural properties, and both are equally informative. But they are not equally simple.

This is problematic. Given gerrymandered second-order relations, even the simplest system can be made to look extraordinarily complex. And systems of arbitrarily complex laws can be made simple if these relations are chosen judiciously. Which should we use when evaluating candidate systems? One option is to use whichever ones yield the simplest formulation of the laws of that system. But since virtually any system can be made to look simple, this move threatens to render the "simplicity" constraint vacuous.

To avoid this result, there need to be constraints on which formulations of the laws are permissible. The permissible formulations are the ones that respect the genuine ordering and distance relations among quantities - i.e. the perfectly 
natural ones. And it's these formulations that should be used when assessing the simplicity of a candidate system.

\subsection{Counterfactuals}

According to Lewis's analysis of counterfactuals, a counterfactual is true at $w$ iff some world at which both the antecedent and the consequent are true is closer to $w$ than any world at which the antecedent is true but the consequent is not (see Lewis $(1973 b)$ ). Closeness of worlds is a matter of objective resemblance - with the relevant aspects of objective resemblance determined by context - and objective resemblance is grounded in the perfectly natural properties and relations.

Consider the following simple counterfactual: if I had let go of the pen, it would have fallen. This claim is true just in case a world at which I let go of the pen and it falls is closer to the actual world than any world at which I let go of the pen and it does not fall. In most contexts, the closest worlds will be ones where the laws of nature are the same (or very similar to) the actual laws, where the history up until the time I drop the pen matches (or very closely matches) the actual history, and so on. So cross-world comparisons are critical in evaluating counterfactual claims, and these cross-world comparisons consist in assessing the degrees of resemblance in various respects among worlds.

Now consider a different counterfactual. Suppose Bob is a very good basketball player. But Bob is small - he is only five feet tall, with a mass of 60kgs and so is not tall enough to play college-level basketball. It seems the following counterfactual is true: "If Bob had been a foot taller, he would have been on the college basketball team." This counterfactual is true just in case a world where Bob is a foot taller and plays college basketball is closer to the actual world than any world where Bob is a foot taller but is not on the basketball team. Now consider two possible candidates for being the closest world, $w_{1}$ and $w_{2}$. At both $w_{1}$ and $w_{2}$, Bob is a foot taller. But at $w_{1}$, Bob has a mass of $500 \mathrm{kgs}$, and so is too heavy to be on the college basketball team. At $w_{2}$, on the other hand, Bob is athletic and his mass is a bit more than his actual mass - say, 75kgs.

Which of these worlds is closer to the actual world? It depends on which second-order relations are relevant when comparing Bob's mass at the actual world to his mass at $w_{1}$ and at $w_{2}$. After all, there are some relations according to which $60 \mathrm{kgs}$ is closer to $500 \mathrm{kgs}$ than to $75 \mathrm{kgs}$, and so $w_{1}$ is closer to the actual world than $w_{2}$. But this is the wrong result. 
Of course, worlds $w_{1}$ and $w_{2}$ differ from the actual world in many ways not just with respect to Bob's mass. For example, since Bob's mass is 500kgs at $w_{1}$, he probably eats a lot more in $w_{1}$ than he does in $w_{2}$ or the actual world. And all of these differences (or at least those deemed relevant by context) must be taken into account when assessing the relative closeness of $w_{1}$ and $w_{2}$. But appeal to these differences does not abrogate the need for perfectly natural second-order relations. In general, the worlds under consideration will differ from the actual world with respect to their quantitative values in lots of ways. If we want to get the right answers when we assess counterfactuals, the closest worlds need to be ones where these quantitative differences are small. So we need to make sure that we assess similarity with respect to the right second-order relations - the perfectly natural ones.

\subsection{Causation}

Here is a rough characterization of Lewis's counterfactual analysis of causation: $C$ causes $E$ iff both $C$ and $E$ occur, and if $C$ had not occurred then $E$ would not have occurred. ${ }^{40}$ The latter condition is analyzed thus: a world where neither $C$ nor $E$ occurs is closer to the actual world than any world where $C$ does not occur and $E$ does occur.

Suppose an enormous asteroid, A, is headed straight for the Earth, and we are powerless to stop it. The asteroid is so massive that if it hits any part of the planet - even if it barely grazes the surface - the impact will be disastrous, and the Earth will be destroyed. Unbelievably, just before A is about to enter the Earth's atmosphere, it collides with another asteroid, B, and the collision deflects A's path just enough so that the Earth is saved from obliteration. Had there been any small variation in circumstances - if A's trajectory had been slightly different, if A had been travelling at a slightly different speed, if A had begun its journey just a few moments earlier or later - then A would not have serendipitously collided with B, and would have gone on to hit the Earth. News anchors across the globe proclaim: "It's a miracle! If A had not collided with $B$, then A would have hit the Earth. The Earth is saved because of an extremely unlikely event!" (Fox News adds: "Proof of God!")

Consider the following causal claim:

(C) A's hitting B caused the Earth to be saved from total annihilation. Given a Lewisian analysis of causation, (C) is true iff a world at which A does not hit $\mathrm{B}$ and the Earth is annihilated by A's impact is closer than any world at which

\footnotetext{
${ }^{40}$ See Lewis ([1973a] 1986c, 159-213) and ([1983] 1999, 43-45).
} 
A does not hit B and the Earth is not annihilated. In the relevant context of utterance, $(C)$ seems true. The closest world at which A does not collide with B is one where A hits the Earth.

Now consider two other worlds, $w_{1}$ and $w_{2}$. Suppose that A's trajectory at $w_{1}$ differs from its trajectory at the actual world by just a small amount - small enough so that it hurtles right past B instead of colliding with it. So at $w_{1}$, A does not collide with B, and A hits the Earth. At $w_{2}$, on the other hand, A's trajectory is radically different from its trajectory at the actual world. At $w_{2}$, A travels in an entirely different direction. So A not only avoids colliding with B at $w_{2}, \mathrm{~A}$ also avoids colliding with the Earth. Furthermore, suppose that $w_{1}$ and $w_{2}$ are relevantly similar to the actual world in other respects.

Is $w_{1}$ or $w_{2}$ closer to the actual world? It depends on which second-order relations are relevant to comparing A's trajectory at the actual world to its trajectory at $w_{1}$ and $w_{2}$. There will be some distance relations according to which A's trajectory at $w_{2}$ is closer to A's actual trajectory than A's trajectory at $w_{1}$. If we use one of these to evaluate objective resemblance, $w_{2}$ will be closer to the actual world than $w_{1}$. So (C) may well be false. But that is the wrong result. If we want to get the right results when it comes to assessing claims of causal dependence, we need to assess similarity with respect to the right second-order relations - the perfectly natural ones.

\section{Minimality}

According to Lewis, the perfectly natural properties and relations do not just characterize the world exhaustively, they also characterize it minimally: "there are only just enough of them to characterise things completely and without redundancy." (Lewis 1986b, 60) ${ }^{41}$ The intuitive idea that the perfectly natural properties are sparse and selective is generally cashed out in terms of supervenience: ${ }^{42,43}$

Minimality: the set of perfectly natural properties does not supervene on any proper subset of them.

\footnotetext{
${ }^{41}$ See also Lewis ([1983] 1999, 12): “The world's universals should comprise a minimal basis for characterising the world completely."

${ }^{42}$ There are various ways to formulate the Minimality constraint. But whichever formulation one chooses the result: if we adopt perfectly natural second-order relations of the sort I've been considering, the perfectly natural properties fail to comprise a minimal supervenience base. ${ }^{43}$ Note that it is a mistake to construe Minimality as part of the definition of perfect naturalness, for reasons given in Sider (1996).
} 
But if some second-order relations are perfectly natural, as I have argued, then Minimality conflicts with the Necessity Assumption, according to which facts regarding the structural features of quantity are necessary (see section (2)). Given an account that employs second-order relations among quantitative properties, the Necessity Assumption entails that if, for instance, two grams mass bears the less than or equal to relation to three grams mass, then this relation holds necessarily; there is no possible world where two grams mass is greater than three grams mass. Likewise, if the sum of relations holds among two grams mass, three grams mass, and five grams mass, then this relation holds necessarily; there is no possible world where two grams mass and three grams mass sum to anything other than five grams mass.

Since these second-order relations are necessary, they supervene on anything whatsoever. ${ }^{44} A$ fortiori, $P$ supervenes on any of the perfectly natural properties and relations. So if $P$ is necessary, and the set of perfectly natural properties and relations includes $P$, then the set violates Minimality.

So which must go, Minimality or the Necessity Assumption? If we drop the Necessity Assumption, we effectively give up the game. For if these relations are not necessary, then it is hard to see how they can play the required roles in accounts of resemblance, duplication, laws, counterfactuals, causation, etc. An example: consider three objects $-a, b$, and $c$ - located in worlds $w_{1}, w_{2}$, and $w_{3}$, respectively. Object $a$ has two grams mass, $b$ has three grams mass, and $c$ has one hundred grams mass. Clearly, $b$ is closer in mass to $a$ than to $c$. (And, if the objects are alike in all other respects, $b$ resembles $a$ more than $c$.) But if the distances between mass quantities may vary from world to world, then there are no non-world-relative facts about the mass-distances between $a, b$, and $c$. And there are no non-world-relative facts about whether $b$ is closer to $a$ or to $c$. Gone is any analysis that employs some notion of closeness between worlds that is tied to the distances between quantities. ${ }^{45}$

\footnotetext{
${ }^{44}$ Roughly, $P$ supervenes on $Q$ iff there is no change in $P$ without a corresponding change in $Q$. If there is never any change in $P$ (because $P$ obtains necessarily), then $P$ supervenes on anything, including $Q$.

${ }^{45}$ One might argue that another way to save Minimality is to claim that some second-order relations are more natural than any others, but none are perfectly natural (thanks to Cody Gilmore who first raised this objection to me). I think this is difficult to sustain. First, the heirarchy of naturalness is supposed to be determined, somehow, by the perfectly natural. But there is nothing about the perfectly natural first-order quantitative properties that "makes" some second-order relations more natural than others. For if there were, an account of quantity need not appeal to second-order relations at all - whether perfectly natural or only somewhat natural - since the "right" second-order relations would be entailed by the first-order quantitative properties alone. Second, the original motivation for adopting a primitive distinction between perfectly natural
} 
A better option is to keep the Necessity Assumption and drop Minimality. One might try to replace Minimality with another claim along similar lines perhaps one might say that the perfectly natural properties and relations comprise a minimal in virtue of base. But this is hardly compulsory. Dropping Minimality does not mean that the perfectly natural properties are thereby abundant and undiscriminating. ${ }^{46}$ They are still sparse and selective, they are still the ones that carve nature at the joints, and they are still the ones that make for objective similarity and difference. ${ }^{47}$

\section{References}

Armstrong, D. M. (1978). Universals and Scientific Realism, Volume II: A Theory of Universals. Cambridge University Press.

Armstrong, D. M. (1988). “Are Quantities Relations? A Reply to Bigelow and Pargetter.” Philosophical Studies 54: 305-316.

Armstrong, D. M. (1989). Universals: An Opinionated Introduction. Colorado: Westview Press.

Bigelow, John and Robert Pargetter (1988). “Quantities.” Philosophical Studies 54: 287-304.

Bigelow, John and Robert Pargetter (1990). Science and Necessity. Cambridge University Press.

Dasgupta, Shamik (2013). “Absolutism vs Comparativism About Quantity.” Oxford Studies in Metaphysics, Vol. 8. Ed. Karen Bennett and Dean Zimmerman. Oxford: Oxford University Press.

Denby, David (2001). “Determinable Nominalism.” Philosophical Studies 102: 297-327.

\footnotetext{
properties and all the rest was the indispensable role perfectly natural properties play in a swath of metaphysical projects. The second-order relations play those very same roles. So to deny them the status of perfectly natural is to give up on many of the reasons for positing perfectly natural properties in the first place.

${ }^{46}$ Nor does allowing necessary relations of this sort violate the Humean proscription of necessary connections - at least not as interpreted by Lewis as "the principle that anything can coexist with anything else, at least provided they occupy distinct spatiotemporal positions." (Lewis 1986b, 88)

${ }^{47}$ Thanks to Cody Gilmore, Boris Kment, Raul Saucedo, Ted Sider, Brad Skow, Kelly Trogdon, and Dean Zimmerman. Special thanks to Chris Meacham for extensive comments and discussion.
} 
Eddon, M. (2007). “Armstrong on Quantities and Resemblance.” Philosophical Studies 136: 385-404.

Eddon, M. (2011). "Intrinsicality and Hyperintensionality." Philosophy and Phenomenological Research 82: 314-336.

Eddon, M. (2013). “Quantitative Properties.” Philosophy Compass.

Ellis, Brian (1966). Basic Concepts of Measurement. Cambridge University Press.

Field, Hartry (1980). Science Without Numbers. Princeton University Press.

Forge, John (1995). “Bigelow and Pargetter on Quantities.” Australasian Journal of Philosophy 73: 594-605.

Hawthorne, John (2006). "Quantity in Lewisian Metaphysics.” In Hawthorne, Metaphysical Essays. Oxford University Press.

Holly, Jan E. (2001). "Pictures of Ultrametric Spaces, the p-adic Numbers, and Valued Fields." American Mathematical Monthly 108: 721-728.

Krantz, D., Luce, R., Suppes, P., \& Tversky, A. (1971). Foundations of Measurement, Volume 1. New York: Academic Press.

Lewis, David (1973a). “Causation.” Journal of Philosophy 70: 556-567, reprinted with postscripts in Lewis 1986c: 159-213.

Lewis, David (1973b). Counterfactuals. Blackwell.

Lewis, David (1983). "New Work for a Theory of Universals.” Australasian Journal of Philosophy 61: 343-377, reprinted in Lewis 1999: 8-55.

Lewis, David (1986a). “Against Structural Universals.” Australasian Journal of Philosophy 64: 25-46, reprinted in Lewis 1999: 78-107.

Lewis, David (1986b). On the Plurality of Worlds. Blackwell.

Lewis, David (1986c). Philosophical Papers, Vol. II. Oxford University Press.

Lewis, David (1991). Parts of Classes. Blackwell.

Lewis, David (1994). “Humean Supervenience Debugged.” Mind 103: 473-490, reprinted in Lewis 1999: 224-247.

Lewis, David (1999). Papers in Metaphysics and Epistemology. Cambridge University Press.

Liggins, David (2003). “On Being Twice as Heavy.” Philosophica Mathematica 11: 203-207. 
Melia, Joseph (1998). “Field's programme: Some interference.” Analysis 58: 63-71. Meyer, Glen (2009). "Extending Hartry Field's Instrumental Account of Applied Mathematics to Statistical Mechanics.” Philosophia Mathematica 17: 273312.

Milne, Peter (1986). "Hartry Field on Measurement and Intrinsic Explanation." British Journal for the Philosophy of Science 37: 340-346.

Mundy, Brent (1987). “The Metaphysics of Quantity.” Philosophical Studies 51: 29-54.

Mundy, Brent (1988). "Extensive Measurement and Ratio Functions." Synthese 75: $1-23$.

Mundy, Brent (1989). “On Quantitative Relationist Theories." Philosophy of Science 56: 582-600.

Sider, Theodore (1996). "Intrinsic Properties." Philosophical Studies 83: 1-27.

Swoyer, Chris (1987). "Metaphysics of Measurement." Measurement, Realism and Objectivity. Ed. John Forge. Dordrecht: D. Reidel Publishing.

Weatherson, Brian (2006). “Asymmetric Magnets Problem." Philosophical Perspectives 20: 479-492.

Wilson, Jessica (2000). "Could Experience Disconfirm the Propositions of Arithmetic?" Canadian Journal of Philosophy 30: 55-84. 